\title{
Aluminium hot extrusion process capability improvement using Six Sigma
}

\author{
Ketan, H. $^{\mathrm{a},{ }^{*},}$ Nassir, M. ${ }^{\mathrm{a}}$ \\ ${ }^{a}$ Mechanical Engineering Department, University of Baghdad, Baghdad, Iraq
}

\begin{abstract}
A B S T R A C T
In this work, the Six Sigma (Define-Measure-Analyse-Improve-Control) DMAIC methodology has been followed to explain the original problem of lowering extrusion process variation and improving the process capability based on the determined Critical Quality Characteristics (CQC). The extrusion process charter worksheet is recognized, a SIPOC (Supplier-Input-Process-OutputCustomer) chart is constructed and a Pareto chart is drawn in the Define phase of the methodology. Measurement data are collected, verifying process stability and verifying process normality by using $\bar{X}-\mathrm{R}$ charts and normality test, respectively. Process capacity index, sigma levels, defects per million opportunities (DPMO) determination in the measure phase using a Histogram. During Analyse phase, Cause and Effect diagram are established to determine their likelihood for the root cause of aluminium extrusion defective products. The suggested solutions are installed in the improve phase. In the Control phase, all tools are applied in the Measure phase are repeated to determine the improvement level. The DMAIC methodology has been applied in the (Ur state company for engineering industries)/(aluminium extrusion factory). The Minitab 16 Software is used for calculations and plot charts. The results for the internal dimension (X1) of the corner section product indicate a reduction in DPMO from 536804 to 185795.09 , sigma level is improved from 1.4 to 2.4 , process yield $(\mathrm{Y})$ is improved from $46 \%$ to $81 \%$, and profit is improved from ID 127.000 to ID 223.000 per $1000 \mathrm{~kg}$.
\end{abstract}

\section{ARTICLE INFO}

Keywords:

Aluminium extrusion process

Six Sigma

DMAIC

Critical quality characteristics Profit

*Corresponding author: hussket@yahoo.com

(Ketan, H.)

Article history:

Received 21 April 2014

Revised 3 September 2015

Accepted 20 October 2015 


\section{References}

[1] García, F.R. (2014). Six Sigma implementation within the building construction industry: A case study of the research building construction, Master of science thesis, University Polytechnic of Valencia, School of Architecture, Valencia, Spain.

[2] Desale, S.V., Deodhar S.V. (2013). Lean Six Sigma principal in construction: A literature review related to conclusions, International Journal of Emerging Technology and Advanced Engineering, Vol. 3, No. 5, 531-535.

[3] El Santty, M.I., Tharwat A.A., Zein Eldin, R.A. (2013). Multi objective Six Sigma methodology: Application on chromojet printing, American Journal of Research Communication, Vol. 1, No. 7, 100-115.

[4] Park, S.H. (2003). Six Sigma for quality and productivity promotion, Productivity Series 32, Asian Productivity Organization, Tokyo, Japan.

[5] Khan, O.H. (2005). A study of critical success factors for Six Sigma implementation in UK organizations, Master of science dissertation, Bradford University, School of Management, UK.

[6] Aksoy, B., Orbak, Â.Y. (2009). Reducing the quantity of reworked parts in a robotic arc welding process, Quality and Reliability Engineering International, Vol. 25, No. 4, 495-512, doi: 10.1002/qre.985.

[7] Thomsett, M.C. (2004). Getting started in Six Sigma, John Wiley \& Sons, Inc., Hoboken, New Jersey, USA.

[8] Farahmand, K., Grajales, J.V.M., Hamidi, M. (2010). Application of Six Sigma to gear box manufacturing, International Journal of Modern Engineering, Vol. 11, No. 1, 12-19.

[9] Basu, R., Wright, J.N. (2003). Quality beyond Six Sigma, Butterworth-Heinemann, Oxford, UK.

[10] Jodi, M.R. (2014). Casting process improvements based on Six Sigma with simulation modelling, Ph.D. thesis, Baghdad University, Mechanical department, Iraq.

[11] Prasad, K.G.D., Subbaiah, K.V., Padmavathi, G. (2012). Application of Six Sigma methodology in an engineering educational institution, International Journal of Emerging Sciences, Vol. 2, No. 2, 222-237.

[12] Mahanti, R., Antony, J. (2005). Confluence of Six Sigma, simulation and software development, Managerial Auditing Journal, Vol. 20, No. 7, 739-762, doi: 10.1108/02686900510611267.

[13] Habibi, A., Rezapour, A. (2015). The ability of implementation of Six Sigma in government hospitals of Babol, $D U$ Journal, Humanities and Social Sciences, Vol. 8, No. 6, 41-63.

[14] Khekale, S.N., Chatpalliwar, A.S., Thakur, N.V. (2010). Minimization of cord wastages in belt industry using DMAIC, International Journal of Engineering Science and Technology, Vol. 2, No. 8, 3687-3694.

[15] Gijo, E.V., Scaria, J., Antony, J. (2011). Application of Six Sigma methodology to reduce defects of a grinding process, Quality and Reliability Engineering International, Vol. 27, No. 8, 1221-1234, doi: 10.1002/qre.1212.

[16] Hung, H.C., Sung, M.H. (2011). Applying Six Sigma to manufacturing processes in the food industry to reduce quality cost, Scientific Research and Essays, Vol. 6, No. 3, 580-591.

[17] Mandahawi, N., Fouad, R.H., Obeidat, S. (2012). An application of customized Lean Six Sigma to enhance productivity at a paper manufacturing company, Jordan Journal of Mechanical \& Industrial Engineering, Vol. 6, No. 1, 103-109.

[18] Gupta, N., Bharti, P.K. (2013). Implementation of Six Sigma for minimizing the defects rate at a yarn manufacturing company, International Journal of Engineering Research and Applications, Vol. 3, No. 2, 1000-1011. 


\section{APEM}

\title{
Izboljšanje postopka vročega ekstrudiranja aluminija z uporabo metode šest sigma
}

\author{
Ketan, H. $^{\mathrm{a},{ }^{,},}$, Nassir, M. $^{\mathrm{a}}$ \\ ${ }^{a}$ Mechanical Engineering Department, University of Baghdad, Baghdad, Iraq
}

\section{POVZETEK}

$\mathrm{V}$ prispevku smo uporabili metodo šest sigma (definiraj-izmeri-analizirajizboljšaj-nadzoruj) za večjo stabilnost tehnološkega procesa in izboljšanje postopka vročega ekstrudiranja aluminija, temelječe na določitvi kritične karakteristike kakovosti (CQC). V fazi definiraj smo izdelali delovni list procesa ekstrudiranja, graf SIPOC (dobavitelj-vhod-proces-izhod-kupec) in Paretov diagram. Zajeli smo podatke merjenj in verificirali stabilnost procesa oziroma testirali normalnost distribucije $\mathrm{z}$ uporabo $\overline{\mathrm{X}}$-R grafov oziroma testa normalnosti. V fazi izmeri smo določili indeks sposobnosti procesa, nivoje sigma in število napak na milijon možnosti (DPMO). Da bi ugotovili temeljni razlog za defektne izdelek smo med fazo analiziraj izdelali diagram vzrok-posledica. Predlagane rešitve smo upoštevali v fazi izboljšaj. V fazi nadzoruj smo ponovili vse aktivnosti iz faze izmeri, da bi ugotovili raven izboljšanja procesa. DMAIC metodologija je bila vpeljana v iraški firmi Ur state company for engineering industries/aluminium extrusion factory. Za preračune in izdelavo grafov je bila uporabljena programska oprema Minitab 16. Rezultati za notranjo dimenzijo (X1) robnega preseka izdelka kažejo zmanjšanje DPMO od 536804 na 185795.09 , nivo sigma se je povečal od 1.4 na 2.4, izkoristek procesa (Y) od $46 \%$ na $81 \%$, dobiček pa od ID 127.000 na ID 223.000 na $1000 \mathrm{~kg}$.

\section{PODATKI O ČLANKU}

Ključne besede:

Proces ekstrudiranja aluminija

Šest sigma

DMAIC

Kritične karakteristike kakovosti Dobiček

*Kontaktna oseba: hussket@yahoo.com

(Ketan, H.)

Zgodovina članka:

Prejet 21. aprila 2014

Popravljen 3. septembra 2015

Sprejet 20. oktobra 2015 